INPLASY

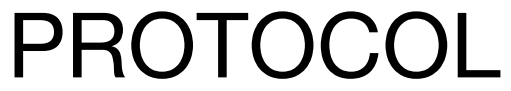

To cite: Liu et al. Efficacy and safety of acupuncture combined with opioid drugs in the treatment of moderate to severe cancer pain: A systematic review and metaanalysis. Inplasy protocol 202180016. doi: 10.37766/inplasy2021.8.0016

Received: 04 August 2021

Published: 04 August 2021

Corresponding author:

Tai-Peng Tan

tanyizhen12345@126.com

Author Affiliation:

Acupuncture department, Heilongjiang Provincial Academy of Traditional Chinese Medicine Sciences, Harbin, China; No.142, Sanfu Street, Xiangfang District, Harbin City, Heilongjiang Province, China

Support: Key Talents of TCM.

Review Stage at time of this submission: Piloting of the study selection process.

Conflicts of interest:

None declared.

\section{Efficacy and safety of acupuncture combined with opioid drugs in the treatment of moderate to severe cancer pain: A systematic review and meta-analysis}

Liu, ZN1; Liu, GF2; Yang, M3; Wang, L4; Zhang, J5; Tan, TP6.

Review question / Objective: Efficacy and safety of acupuncture combined with opioid drugs in the treatment of moderate to severe cancer pain: A systematic review and meta-analysis.

Condition being studied: Pain is one of the most common and difficult to control symptoms of cancer patients, and it is a major factor affecting the quality of life of patients. At present, the treatment of moderate to severe cancer pain is mainly based on opioid therapy. As the degree of pain increases, the amount of opioids continues to increase. Long-term use of opioids is extremely prone to adverse reactions such as nausea and vomiting, dizziness, headache, and constipation. In recent years, there have been more and more researches on acupuncture treatment of cancer pain, and the quality of research has gradually improved. This article aims to use a systematic review method to understand the efficacy and safety of acupuncture combined with opioids in the treatment of moderate to severe cancer pain.

INPLASY registration number: This protocol was registered with the International Platform of Registered Systematic Review and Meta-Analysis Protocols (INPLASY) on 04 August 2021 and was last updated on 04 August 2021 (registration number INPLASY202180016).

\section{INTRODUCTION}

Review question / Objective: Efficacy and safety of acupuncture combined with opioid drugs in the treatment of moderate to severe cancer pain: A systematic review and meta-analysis.

Condition being studied: Pain is one of the most common and difficult to control 
symptoms of cancer patients, and it is a major factor affecting the quality of life of patients. At present, the treatment of moderate to severe cancer pain is mainly based on opioid therapy. As the degree of pain increases, the amount of opioids continues to increase. Long-term use of opioids is extremely prone to adverse reactions such as nausea and vomiting, dizziness, headache, and constipation. In recent years, there have been more and more researches on acupuncture treatment of cancer pain, and the quality of research has gradually improved. This article aims to use a systematic review method to understand the efficacy and safety of acupuncture combined with opioids in the treatment of moderate to severe cancer pain.

\section{METHODS}

Participant or population: Patients clearly diagnosed with moderate to severe cancer pain, age, gender, course of disease and other factors are not limited.

Intervention: Acupuncture and opioid drugs was the main intervention.

Comparator: Acupuncture, Opioid drugs or some other means of treatment.

Study designs to be included: Randomised controlled trial will be included.

Eligibility criteria: Patients clearly diagnosed with moderate to severe cancer pain, age, gender, course of disease and other factors are not limited.Randomised controlled trial will be included.

Information sources: We will search the randomized controlled trails (RCT) literatures of WNA for moderate to severe cancer pain in 8 electronic databases, including 4 English databases [PubMed, EMBASE, the Cochrane Central Register of Controlled Trials (Cochrane Library) and Web of Science] and 4 Chinese databases [Chinese National Knowledge Infrastructure(CNKI), Chinese VIP Information, Wanfang Database, and Chinese Biomedical Literature Database
(CBM)],from their inception to June 2021, to identify and retrieve all randomised controlled trials, describing the use of WNA for treatment of moderate to severe cancer pain. We also contacted the authors, searching for experimental data, clinical research registration, or other related grey literature.

Main outcome(s): The course of treatment, the dose and type of opioids, and the course of the disease are related to the effective rate, cure rate, adverse reaction rate, NRS score, KPS score and QOL score.

Quality assessment / Risk of bias analysis: Two reviewers will independently assesses the quality of the selected studies according to the Cochrane Collaboration's tool for randomized controlled trials. Items will be evaluated in three categories: Low risk of bias, unclear bias and high risk of bias. The following characteristics will be evaluated: Random sequence generation (selection Bias)Allocation concealment (selection bias) Blinding of participants and personnel ((performance bias) Incomplete outcome data (attrition bias) Selective reporting (reporting bias) Other biases Results from these questions will be graphed and assessed using Review Manager 5.3.

Strategy of data synthesis: Two authors will independently extract data. Any disagreement will be resolved by discussion until consensus is reached or by consulting a third author. The following data will be extracted:author, year of publication, country where the study was conducted, study period, original inclusion criteria, total number of people included in the study, group, interventions, sample size, age (years), gender, course of the disease, course of treatment.

Subgroup analysis: Once individual studies may consist of multiple treatment group, subgroup analysis will be performed to explain heterogeneity if possible. Factors such as following will be considered: Patients characteristics (age, sex), course of the disease, different scales, frequency of therapy, type and dose of opioids. 
Sensitivity analysis: In order to ensure the stability of the outcome index results, the sensitivity analysis of each outcome index was carried out.

Country(ies) involved: China.

Other relevant information: (1)Zheng-Nan Liu: The First Affiliated Hospital of Guangzhou University of Traditional Chinese Medicine, Guangzhou, China. (2)Gao-Feng Liu: Graduate Faculty, Tianjin University of Traditional Chinese Medicine, Tianjin, China. (3)Min Yang: Dermatology department, Qujiang District Hospital of Traditional Chinese Medicine, Quzhou, China. (4)Lin Wang: The First Affiliated Hospital of Guangzhou University of Traditional Chinese Medicine, Guanzhou, China. (5)Jing Zhang: Acupuncture department, Heilongjiang Provincial Academy of Traditional Chinese Medicine Sciences, Harbin, China. (6)Tai-Peng Tan: Acupuncture department, Heilongjiang Provincial Academy of Traditional Chinese Medicine Sciences, Harbin, China.

Keywords: acupuncture, opioid drugs, moderate to severe cancer pain, RCT, meta-analysis.

Contributions of each author:

Author 1 - Zheng-Nan Liu.

Author 2 - Gao-Feng Liu.

Author 3 - Min Yang.

Author 4 - Lin Wang.

Author 5 - Jing Zhang.

Author 6 - Tai-Peng Tan. 